\title{
Can Quality Improvement Tools Overcome the Translational Roadblock - the Vital Influence of the Researcher
}

\author{
Serge Marbacher ${ }^{1,2}$
}

Received: 28 January 2017 / Accepted: 30 January 2017 /Published online: 24 February 2017

(C) Springer Science+Business Media New York 2017

Both special issues "Challenges and Controversies in Translational Stroke Research, Part 1 and 2" provide an excellent overview of the methodological challenges encountered in the various stages of planning, implementation, and evaluation of preclinical stroke studies. The topics discussed do not apply solely to the field of stroke research, but to many other fields of translational science as well. This collection of articles by highly informed scientists clearly illustrates that a multitude of approaches and measures is needed to address bias in preclinical research in order to increase the translation of knowledge from basic science to clinical practice.

Over the past decade, preclinical researchers have learned just how prone to bias each stage of their experimental work is. Due to increased awareness of widespread methodological bias and obvious translational roadblocks in stroke research, checklists and guidelines have been developed to support basic researchers in the design, execution, analysis, interpretation, and reporting of animal experiments. Not only have guidelines been published to improve the general design and reporting of preclinical animal studies, the methodology of translational research as such has also been questioned. Novel approaches have been proposed to minimize bias and improve translational success. These strategies range from engaging multiple laboratories in preclinical trials [1], to increasing awareness of the importance of sex, age, and

Serge Marbacher

serge.marbacher@ksa.ch; neurosurgery@ksa.ch

Kantonsspital Aarau, Aarau, Switzerland

2 University of Bern, Bern, Switzerland strain-related differences [2, 3], recognizing biological and methodological variances [4], the impact of diseasespecific comorbidities [5] and anesthetics [6] on measured endpoints, and using proper outcome modalities [7-9] in experimental stroke research.

One of the main goals of introducing guidelines for designing, executing, and reporting of in vivo animal experiments was to enable better systematic reviews and meta-analyses of preclinical study publications. Highquality systematic reviews of animal experiments will most likely improve scientific quality, as well as decision-making on whether or not to start a clinical trial in the first place. Although rigor in implementation of standards in animal studies is probably the best way to achieve reproducible results - and facilitate systematic reviews and meta-analysis - one must keep in mind that experiments conducted under highly standardized conditions may represent a driver of poor reproducibility (producing results with little external validity) [10].

Multilaboratory studies would likely contribute to the heterogenization of experimental conditions, presumably increase external validity and, as a result, the predictive value of preclinical research. Community-based feedback regarding multicenter preclinical randomized trials indicated a high level of acceptance for the currently defined potential frameworks and guidelines [1]. However, the survey also revealed that there is room for improvement in quality assurance. One third of participants, for example, did not see a need for blinding and/or randomization, and less than half of researchers considered arterial blood gases/pH monitoring, or investigating at least two species (STAIR recommendations) to be necessary [1].

This is an important finding and corroborates concerns that implementing frameworks and guidelines proposed 
by selected experts and renowned individuals can be challenging within the research community base. Reporting guidelines (e.g., ARRIVE), which represent a cornerstone of efforts to reduce bias in animal research, are largely ignored [11]. Less than half of authors publishing in journals which conform to guidelines have even heard of them [12]. It is therefore of utmost importance to raise awareness of tools available to improve planning, reporting, and analyzing preclinical stroke research (Table 1) among grass root researchers, and that journal editors and reviewers feel responsibility for enforcing adherence to guidelines.

In addition to stepping up efforts to rigorously implement existing tools in order to reduce risks of bias and advance the quality of animal studies, further research is needed to improve the construct validity of animal models and to understand underlying causes of poor external validity [12]. However, any measure is only as good as its acceptance by the broad research community. Educating young researchers in good
Table 1 Tools to improve planning, reporting, and analyzing preclinical (stroke) research

\begin{tabular}{|c|c|}
\hline Tool (reference) & Description (hyperlink) \\
\hline STAIR $[13,14]$ & $\begin{array}{l}\text { Stroke Therapy Academic Industry Roundtable. Collaborative effort by academics and } \\
\text { industry to advance the field of stroke research by introducing recommendations and } \\
\text { guidelines for evaluation of preclinical neuroprotective drugs. }\end{array}$ \\
\hline STEPS $[15,16]$ & $\begin{array}{l}\text { Stem cell Therapies as an Emerging Paradigm in Stroke. Initiative for developing stem cell } \\
\text { therapy for stroke. Some overlap with the STAIR recommendations. }\end{array}$ \\
\hline RIGOR [17] & $\begin{array}{l}\text { Recommendations and guidelines to improve the quality of preclinical research through } \\
\text { rigorous study design and transparent reporting data from experimental studies. } \\
\text { (http://www.ninds.nih.gov/funding/transparency_in_reporting_guidance.pdf) }\end{array}$ \\
\hline EDA [18] & $\begin{array}{l}\text { Experimental Design Assistant. Software designed to support researchers in the planning of } \\
\text { animal experiments, ensuring robust study design, and reliable and reproducible findings. } \\
\text { (https://eda.nc3rs.org.uk/) }\end{array}$ \\
\hline DEPART [19] & $\begin{array}{l}\text { Design and Execution of Protocols for Animal Research and Treatment. A checklist to } \\
\text { consult prior to starting experiments. Includes considerations that should be made during } \\
\text { the design and execution of animal experiments. }\end{array}$ \\
\hline ARRIVE [20] & $\begin{array}{l}\text { Animal Research: Reporting of In Vivo Experiments. Developed as part of an NC3Rs } \\
\text { (National Centre for the Replacement, Refinement and Reduction of Animals in } \\
\text { Research) initiative to improve the design, analysis, and reporting of research using } \\
\text { animals. More commonly used than the GSPC guidelines. (https://www.nc3rs.org. } \\
\text { uk/arrive-guidelines) }\end{array}$ \\
\hline GSPC [21] & $\begin{array}{l}\text { Gold Standard Publication Checklist. Initiative to optimize the design and execution of new } \\
\text { animal experiments and to increase the quality of reporting of completed animal studies. } \\
\text { Some overlap with the ARRIVE guidelines but certain items (housing conditions, } \\
\text { nutrition) are given in greater detail. More focused on the planning, design, and execution } \\
\text { of animal experiments rather than its reporting. }\end{array}$ \\
\hline CAMARADES & $\begin{array}{l}\text { Collaborative Approach to Meta-Analysis and Review of Animal Data from Experimental } \\
\text { Studies. Developed for evaluating candidate stroke drugs. This platform provides a } \\
\text { support framework for groups involved in the systematic review and meta-analysis of data } \\
\text { from experimental animal studies in general. It aims to act as a central hub for data sharing } \\
\text { and act as repository for completed reviews. (http://www.den.ed.ac.uk/camarades/default. } \\
\mathrm{htm} \text { ) }\end{array}$ \\
\hline SYRCLE & $\begin{array}{l}\text { SYstematic Review Centre for Laboratory animal Experimentation. Focuses on the } \\
\text { execution of systematic reviews of animal studies towards more evidence-based transla- } \\
\text { tional medicine. SYRCLE develops training programs, methodological tools, and guide- } \\
\text { lines to support researchers who plan to perform a systematic review of preclinical pub- } \\
\text { lications. (https://www.radboudumc. } \\
\text { nl/Research/Organisationofresearch/Departments/cdl/SYRCLE) }\end{array}$ \\
\hline P3PT $[22,23]$ & $\begin{array}{l}\text { Multicenter Phase III Preclinical Trials. Concept to enhance the predictive value of } \\
\text { preclinical stroke research. Confirmative research conducted by collaborating centers to } \\
\text { rigorously assess the safety and efficacy of a given concept (preclinical phase I and II } \\
\text { studies) before the initiation of early stage clinical trials. }\end{array}$ \\
\hline Multi-PART & $\begin{array}{l}\text { Multicentre Preclinical Animal Research Team. An international collaborative approach to } \\
\text { overcoming the translational roadblocks in neuroprotection and neuroregeneration } \\
\text { research by establishing a platform for international multicenter preclinical stroke trials. } \\
\text { (http://www.dcn.ed.ac.uk/multipart) }\end{array}$ \\
\hline
\end{tabular}


laboratory practices, encouraging respect for their wide applicability (in small research laboratories with fewer personnel and financial resources), as well as the suitability of suggested measures for daily use will probably play a crucial role in the battle to overcome translational roadblocks.

\section{Compliance with Ethical Standards}

Conflict of Interest None.

\section{References}

1. Boltze J, Wagner DC, Henninger N, Plesnila N, Ayata C. Phase iii preclinical trials in translational stroke research: community response on framework and guidelines. Transl Stroke Res. 2016;7: 241-7.

2. Ahnstedt H, McCullough LD, Cipolla MJ. The importance of considering sex differences in translational stroke research. Transl Stroke Res. 2016;7:261-73.

3. Becker KJ. Strain-related differences in the immune response: relevance to human stroke. Transl Stroke Res. 2016;7:303-12.

4. Kent TA, Mandava P. Embracing biological and methodological variance in a new approach to pre-clinical stroke testing. Transl Stroke Res. 2016;7:274-83.

5. Ergul A, Hafez S, Fouda A, Fagan SC. Impact of comorbidities on acute injury and recovery in preclinical stroke research: focus on hypertension and diabetes. Transl Stroke Res. 2016;7:248-60.

6. Hoffmann U, Sheng H, Ayata C, Warner DS. Anesthesia in experimental stroke research. Transl Stroke Res. 2016;7:358-67.

7. Hommel M, Detante O, Favre I, Touze E, Jaillard A. How to measure recovery? Revisiting concepts and methods for stroke studies. Transl Stroke Res. 2016;7:388-94.

8. Muir KW, Macrae IM. Neuroimaging as a selection tool and endpoint in clinical and pre-clinical trials. Transl Stroke Res. 2016;7: 368-77.

9. Jolkkonen J, Kwakkel G. Translational hurdles in stroke recovery studies. Transl Stroke Res. 2016;7:331-42.

10. Richter SH, Garner JP, Wurbel H. Environmental standardization: cure or cause of poor reproducibility in animal experiments? Nat Methods. 2009;6:257-61.
11. Baker D, Lidster K, Sottomayor A, Amor S. Two years later: journals are not yet enforcing the arrive guidelines on reporting standards for pre-clinical animal studies. PLoS Biol. 2014;12: e1001756.

12. Reichlin TS, Vogt L, Wurbel H. The researchers' view of scientific rigor-survey on the conduct and reporting of in vivo research. PLoS One. 2016;11:e0165999.

13. Stroke Therapy Academic Industry R. Recommendations for standards regarding preclinical neuroprotective and restorative drug development. Stroke. 1999;30:2752-8.

14. Saver JL, Albers GW, Dunn B, Johnston KC, Fisher M, Consortium SV. Stroke therapy academic industry roundtable (stair) recommendations for extended window acute stroke therapy trials. Stroke. 2009;40:2594-600.

15. Stem Cell Therapies as an Emerging Paradigm in Stroke P. Stem cell therapies as an emerging paradigm in stroke (steps): bridging basic and clinical science for cellular and neurogenic factor therapy in treating stroke. Stroke. 2009;40:510-5.

16. Savitz SI, Chopp M, Deans R, Carmichael T, Phinney D, Wechsler $\mathrm{L}$, et al. Stem cell therapy as an emerging paradigm for stroke (steps) ii. Stroke. 2011;42:825-9.

17. Lapchak PA, Zhang JH, Noble-Haeusslein LJ. Rigor guidelines: escalating stair and steps for effective translational research. Transl Stroke Res. 2013;4:279-85.

18. Cressey D. Web tool aims to reduce flaws in animal studies. Nature. 2016;531:128.

19. Smith MM, Clarke EC, Little CB. Considerations for the design and execution of protocols for animal research and treatment to improve reproducibility and standardization: "Depart well-prepared and arrive safely". Osteoarthritis Cartilage. 2016.

20. Kilkenny C, Browne WJ, Cuthill IC, Emerson M, Altman DG. Improving bioscience research reporting: the arrive guidelines for reporting animal research. PLoS Biol. 2010;8:e1000412.

21. Hooijmans CR, Leenaars M, Ritskes-Hoitinga M. A gold standard publication checklist to improve the quality of animal studies, to fully integrate the three rs, and to make systematic reviews more feasible. Altern Lab Anim. 2010;38:167-82.

22. Dirnagl U, Fisher M. International, multicenter randomized preclinical trials in translational stroke research: it's time to act. J Cereb Blood Flow Metab. 2012;32:933-5.

23. Bath PM, Macleod MR, Green AR. Emulating multicentre clinical stroke trials: a new paradigm for studying novel interventions in experimental models of stroke. Int J Stroke. 2009;4:471-9. 